\title{
Correlation between bulk material defects and spectroscopic response in cadmium zinc telluride detectors
}

\author{
B. H. Parker ${ }^{\mathrm{a}}$, C. M. Stahle ${ }^{\mathrm{a}}$, S. D. Barthelmy ${ }^{\mathrm{a}, \mathrm{b}}$, A. M. Parsons, , J. Tueller, ${ }^{\mathrm{a}}$ \\ J. T. Van Sant ${ }^{\mathrm{a}}$, B. F. Munoz ${ }^{\mathrm{a}, \mathrm{c}}$, S. J. Snodgrass ${ }^{\mathrm{a}, \mathrm{d}}$, R. E. Mullinix ${ }^{\mathrm{a}}$ \\ aNASA Goddard Space Flight Center, Greenbelt, MD 20771, USA \\ 'Universities Space Research Associates, Greenbelt MD, 20771, USA \\ 'Unisys Corporation, Greenbelt MD, 20771, USA \\ ${ }^{d}$ Raytheon STX, Greenbelt MD, 20771, USA
}

\begin{abstract}
One of the critical challenges for large area cadmium zinc telluride ( $\mathrm{CdZnTe}$ ) detector arrays is obtaining material capable of uniform imaging and spectroscopic response. Two complementary nondestructive techniques for characterizing bulk CdZnTe have been developed to identify material with a uniform response. The first technique, infrared transmission imaging, allows for rapid visualization of bulk defects. The second technique, $x$-ray spectral mapping, provides a map of the material spectroscopic response when it is configured as a planar detector. The two techniques have been used to develop a correlation between bulk defect type and detector performance. The correlation allows for the use of infrared imaging to rapidly develop wafer mining maps. The mining of material free of detrimental defects has the potential to dramatically increase the yield and quality of large area CdZnTe detector arrays.
\end{abstract}

Keywords: CdZnTe, Infrared Transmission Imaging, Spectral Mapping

\section{INTRODUCTION}

Cadmium zine telluride ( $\mathrm{CdZnTe}$ ) has become a key detector technology for hard $\mathrm{x}$-ray and gamma ray astronomy. ${ }^{1.2,3}$ One class of astronomy instruments will use large area single focal plane array detectors in conjunction with a focusing optic. ${ }^{4}$ In addition, the medical imaging community is interested in developing large area $\mathrm{CdZnTe}$ detector arrays. ${ }^{5}$ Because of this common interest, Goddard Space Flight Center and the University of Arizona are collaborating to produce 64 by 64 pixel detectors with $380 \mu \mathrm{m}$ pitch pixels. The detectors require 26.9 by $26.9 \mathrm{~mm}$ specimens with thickness up to $2 \mathrm{~mm}$. One challenge for these arrays is to obtain CdZnTe specimens of this size with uniform spectroscopic response. In this paper, we will describe two complementary nondestructive techniques for characterizing CdZnTe. The first technique, infrared (IR) transmission imaging, allows for rapid visualization of bulk defects such as cracks, grain and twin boundaries decorated with tellurium inclusions, voids and dispersed tellurium inclusions. The second technique, $\mathbf{x}$-ray spectral mapping, involves configuring the bulk material as a planar detector and exposing an area to a collimated beam of photons of variable energy. This interrogation is repeated in a scanning process to create a spatial map of spectral response. The two techniques have been used to develop a correlation between bulk material defects and detector response. Based on this correlation, a wafer-scale mapping program was implemented with $\mathrm{eV}$ Products. Polished wafer slices provided by $\mathrm{eV}$ Products were IR imaged to identify areas with the best potential for producing detectors with a uniform spectral response. The selected areas were then cut from the wafers and their spectroscopic response was mapped.

\section{EXPERIMENT}

\subsection{Infrared Transmission Imaging}

Numerous investigators have used IR transmission imaging to document the distribution of bulk defects in $\mathrm{CdZnTe}{ }^{6.7 .8}$ Most IR transmission imaging is performed using silicon CCD cameras operating at wavelengths just beyond the visible range. These setups typically use an incandescent light as a radiation source. Instead of a silicon CCD camera, we use an 


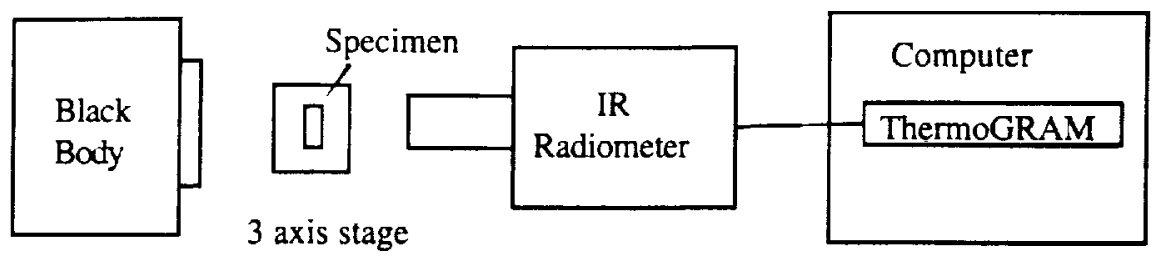

Figure I. IR transmission setup.

Inframetrics Model $600 \mathrm{IR}$ radiometer that operates in the 8 to 12 micrometer bandwidth and has a liquid nitrogen cooled (77 ${ }^{\circ} \mathrm{K}$ ) mercury cadmium telluride ( $\mathrm{HgCdTe}$ ) detector. A large area calibrated black body set at $70^{\circ} \mathrm{C}$ is used as the source of radiation. The setup, which is shown in Figure 1, includes a 3-axis stage for specimen manipulation and a PC based Inframetrics ThermaGRAM ${ }^{\mathrm{TM}}$ data acquisition system for image acquisition, averaging and storage. A wide range of lenses, including a microscope objective, is employed to optimize defect imaging. Figure 2 is a typical IR transmission image of a 26.9 by 26.9 by $2 \mathrm{~mm}$ thick CdZnTe specimen from eV Products. This image, which is the mosaic of six images obtained using an optic with a 16.3 by $12.7 \mathrm{~mm}$ field of view, was selected because it shows the types of bulk defects historically encountered in material obtained from eV Products. These defects include grain and twin boundaries decorated with tellurium inclusions and pipe-like voids.

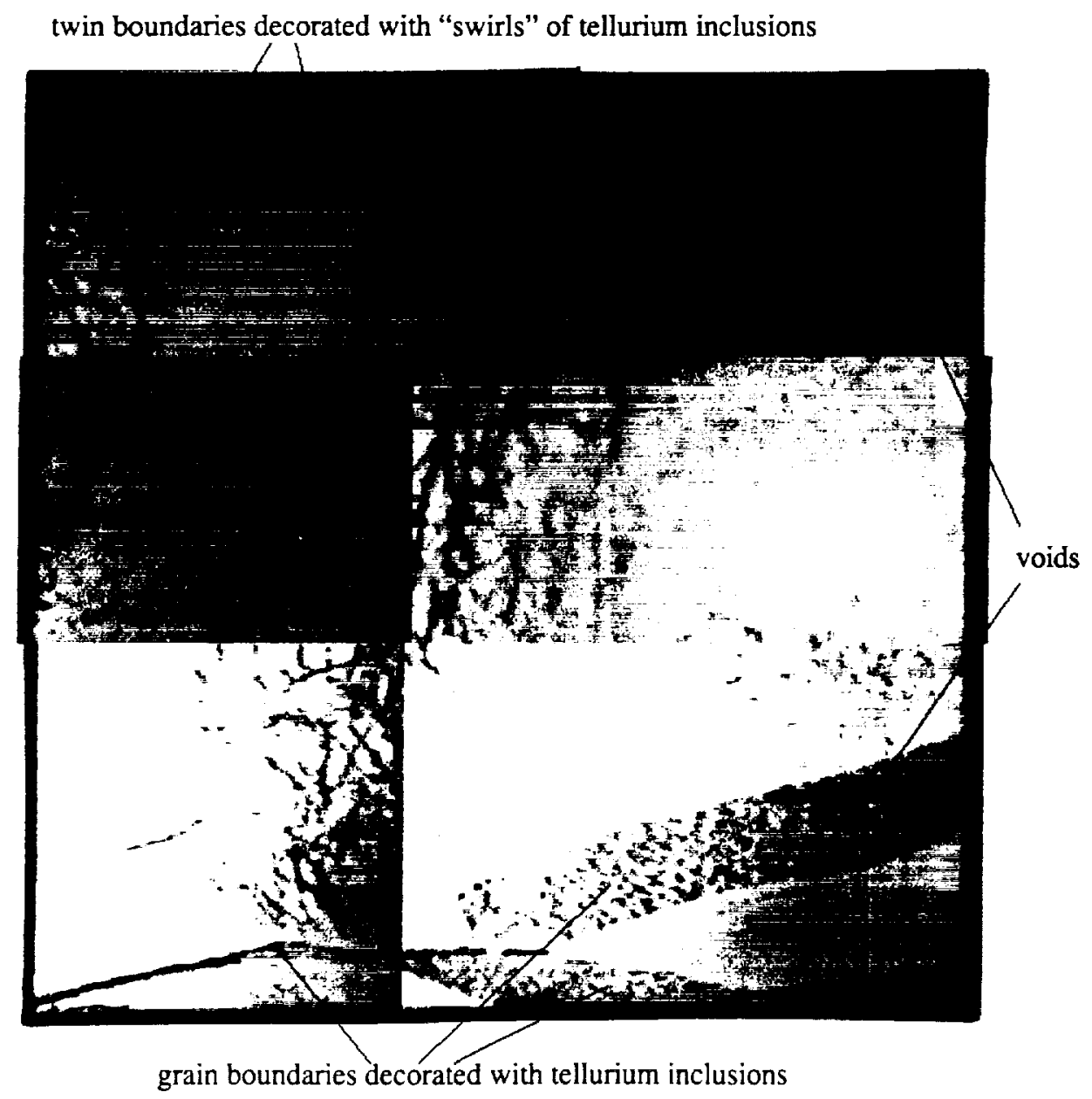

Figure 2. IR transmission image. 


\subsection{X-ray Spectral Mapping}

Several investigators have developed systems to perform spatial mapping of electrical properties of $\mathrm{CdZnTe}$ that are deemed critical to detector performance. The most commonly measured properties are leakage current, which is an indication of material resistivity, ${ }^{8.9}$ and the electron and hole mobility-lifetime products, which are either directly measured using an alpha particle source or inferred from alpha particle pulse height measurements. ${ }^{9}$ However, the most useful map would directly measure the material response to monochromatic radiation with energies that span the range of interest. This typically means using a radioactive isotope as a photon source. In addition, when mapping the material uniformity, the resolution (spot size and step size) should be on a sub-pixel scale. As noted earlier, the arrays currently under development at Goddard Space Flight Center and the University of Arizona have $380 \mu \mathrm{m}$ pitch pixels. Hence, it is desirable to map the material response using $100 \mu \mathrm{m}$ resolution. Collimators of this size dramatically reduce the photon flux from an isotope source, and hence mapping large areas at this resolution is time intensive.

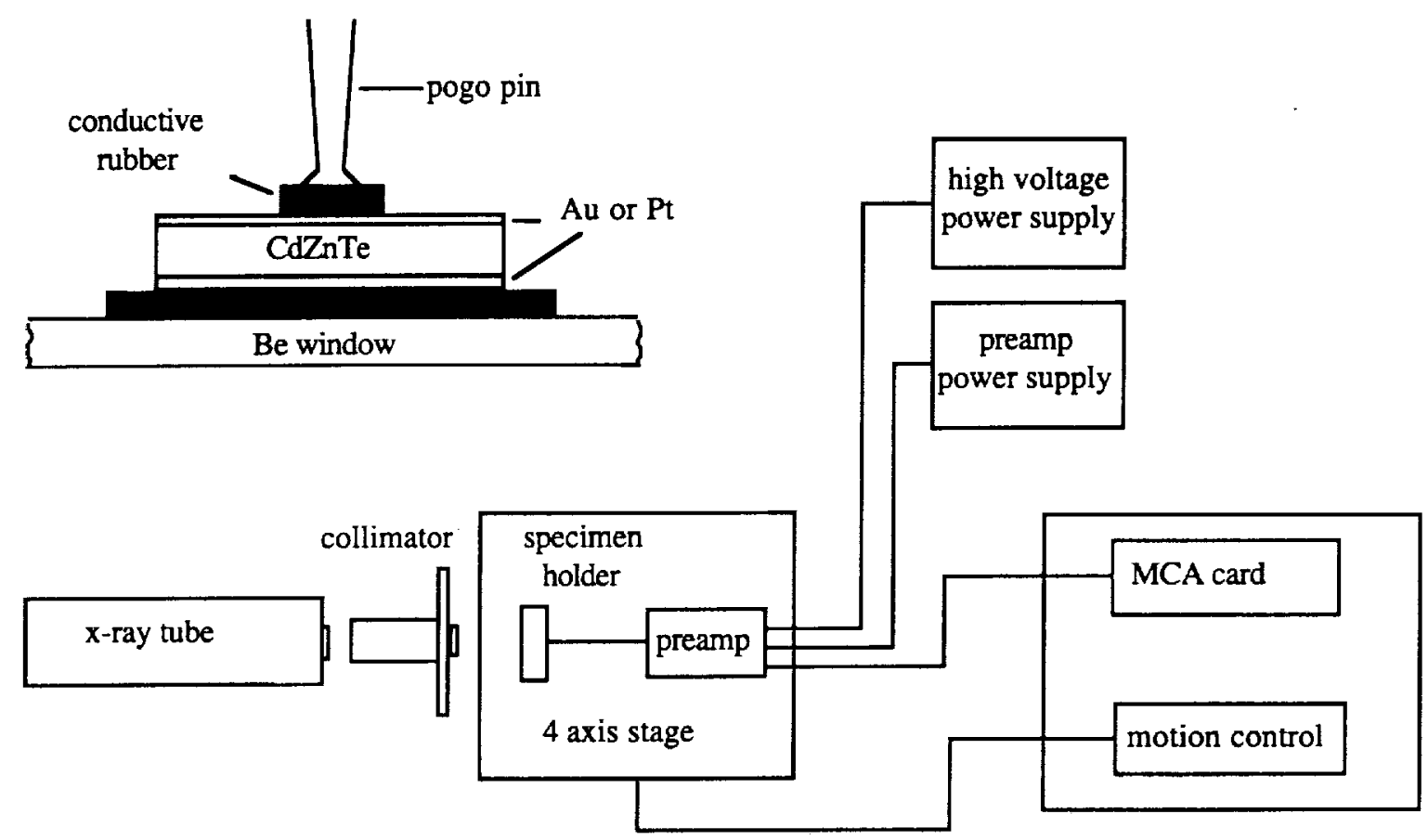

Figure 3. X-ray spectral mapping setup.

With speed in mind, we selected a $160 \mathrm{kV}$ microfocus $\mathrm{x}$-ray tube instead of radioactive isotopes as a source of photons. The $\mathrm{x}$ ray tube produces an extremely high number of photons even when the source is collimated to beam diameters as small as $100 \mu \mathrm{m}$. The limit on the photon flux is not determined by the tube, but by pulse pileup. The high photon count rates ( $1 \times 10^{5}$ counts per second) allow for spectrum accumulation times as low as three seconds. The spectrum from the $\mathrm{x}$-ray tube, which has a tungsten target, consists of bremsstrahlung as well as the characteristic $\mathrm{x}$-ray lines of the tungsten target. Conveniently, the tungsten k-alpha line has an energy of approximately $59.3 \mathrm{keV}$, which is near the midpoint of the energy range of proposed hard $\mathrm{x}$-ray astronomy missions. With three second counting times, we can acquire a spectrum with approximately 1000 counts in the peak channel, i.e., the tungsten $k$-alpha line.

For spectrum acquisition, the CdZnTe specimen is setup as a planar detector. Depending on the specimen size, the anode side of the detector may be a single planar contact or several large pixels with a guard ring. The pixels, whose size is based on 
leakage current noise, are typically 15 by $15 \mathrm{~mm}$ or smaller for high pressure Bridgeman $\mathrm{CdZnTe}$. The nominal bias is 100 Volts per $\mathrm{mm}$ of material thickness. We have 500,250 and $100 \mu \mathrm{m}$ diameter collimators. The biased specimen is moved relative to the collimated $x$-ray beam using a 4 axis motorized stage with a PC based controller. A simple pulse height analysis system is used to acquire the spectra. The multi-channel analyzer (MCA) and the motion are both controlled using LabView ${ }^{\mathrm{TM}}$. The LabView ${ }^{\mathrm{TM}}$ program is also used to extract and store data from the acquired spectra. Plots of peak channel, counts in the peak channel and the ratio of the peak channel counts to background ( $40 \mathrm{keV}$ ) counts are generated. The entire system configuration is shown in Figure 3, and a typical spectrum from the tube is shown in Figure 4.

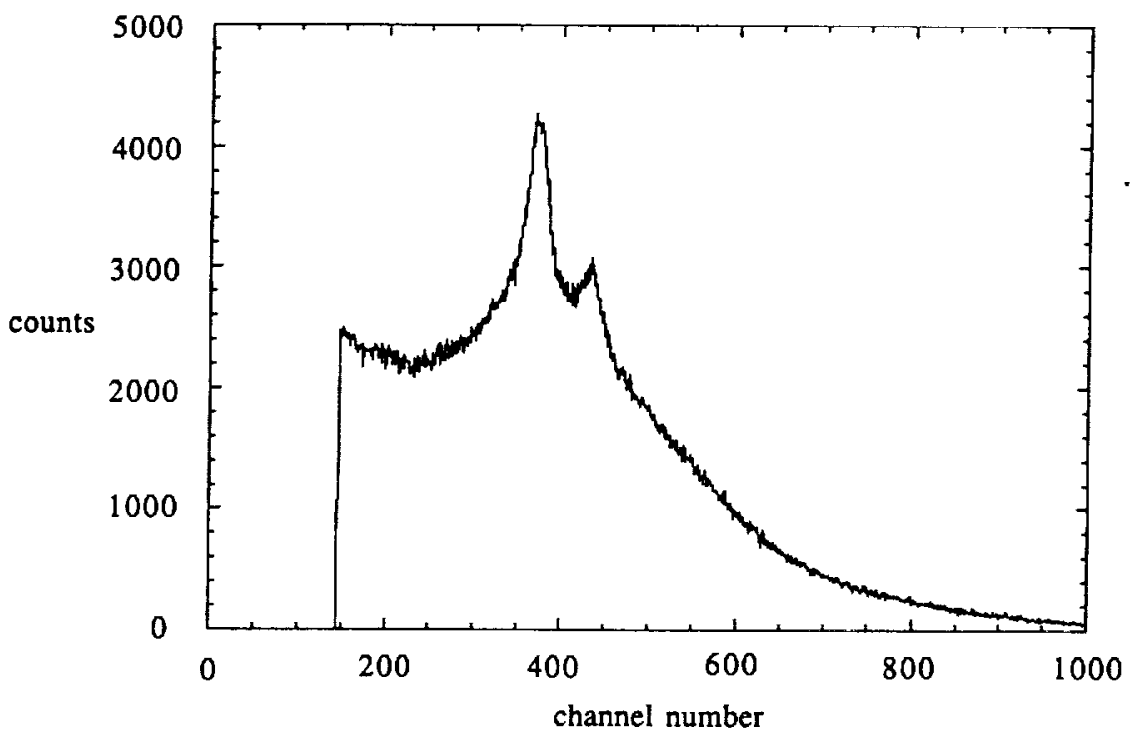

Figure 4. Typical spectrum from $\mathrm{x}$-ray tube as detected by a planar CdZnTe detector.

\section{RESULTS}

\subsection{Correlation Data}

One goal of the $\mathrm{CdZnTe}$ characterization work is to establish a correlation between IR transmission images and spectral maps. This correlation would be used to provide crystal growers with feedback on the types and population densities of bulk defects that are deleterious to detector performance. The initial correlation work was performed on three $\mathrm{eV}$ Products discriminator grade 15 by 15 by $2 \mathrm{~mm} \mathrm{CdZnTe} \mathrm{specimens} \mathrm{from} \mathrm{our} \mathrm{program} \mathrm{to} \mathrm{develop} \mathrm{strip} \mathrm{detectors.}{ }^{10}$ Figure 5 shows an IR transmission image and the corresponding spectral map obtained from one specimen. For the spectral map, the specimen had approximately 14 by $14 \mathrm{~mm}$ planar gold contacts on both sides. A $100 \mu \mathrm{m}$ collimator was used, and the specimen was moved in $250 \mu \mathrm{m}$ steps. These images show a correlation between poor detector response and a grain boundary decorated with tellurium inclusions. The same results were seen in the remaining two specimens.

Similar results were obtained using a $500 \mu \mathrm{m}$ collimator and a $500 \mu \mathrm{m}$ step size on a set of nine 26.9 by 26.9 by $2 \mathrm{~mm}$ $\mathrm{CdZnTe}$ specimens also obtained from $\mathrm{eV}$ Products. The larger collimator and step size were used to reduce the mapping time. For these specimens, four large pixels $(12.6$ by $12.6 \mathrm{~mm}$ ) were used and each pixel was mapped independently. While mapping a single pixel, all four pixels and a guard-ring were biased. Figure 6 shows a representative correlation. Again, tellurium decorated grain boundaries result in a corresponding poor detector response. Similar results were obtained from the remaining specimens and based on the mapping results, only one of the nine specimens was accepted by Goddard Space Flight Center for a focal plane detector. 
Based on the spectral maps and IR transmission images from the two sets of specimens, we developed a correlation between bulk defects visible in the IR and detector performance. The data showed a strong correlation between poor detector response and tellurium decorated grain boundaries. This finding is consistent with results previously reported in the literature. ${ }^{6.7}$

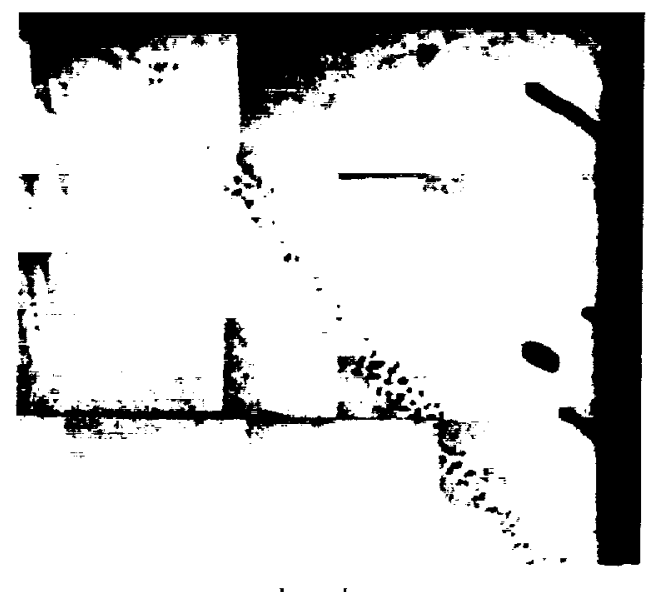

$1 \mathrm{~mm}$

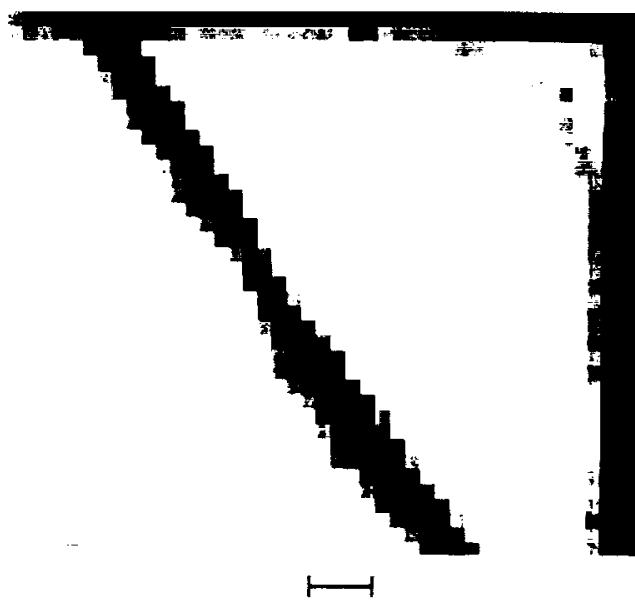

$1 \mathrm{~mm}$
3000

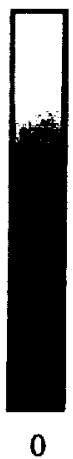

Figure 5. (a) IR transmission image of a portion of eV Products specimen 1182-17 showing a grain boundary decorated with tellurium inclusions. (b) $\mathrm{X}$-ray spectral map of eV Products specimen 1182-17 showing poor spectral response in the area of the decorated grain boundary.

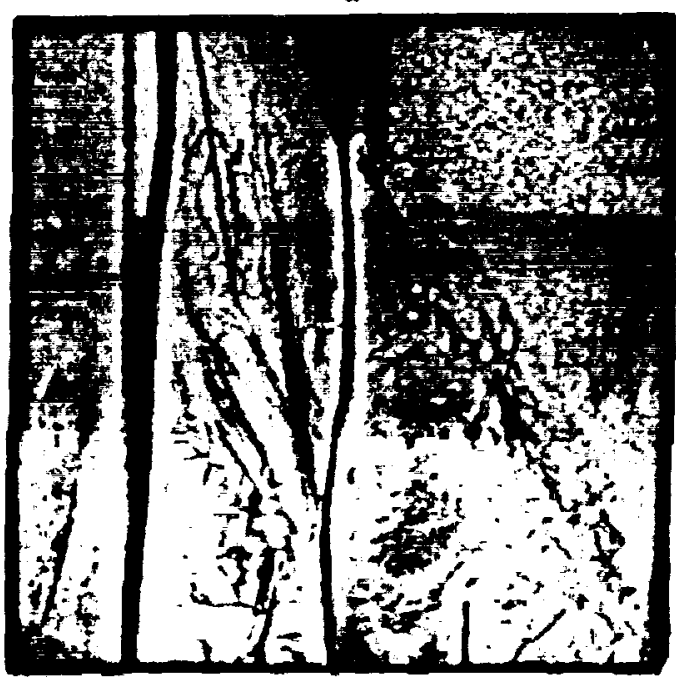

$1 \mathrm{~cm}$ b
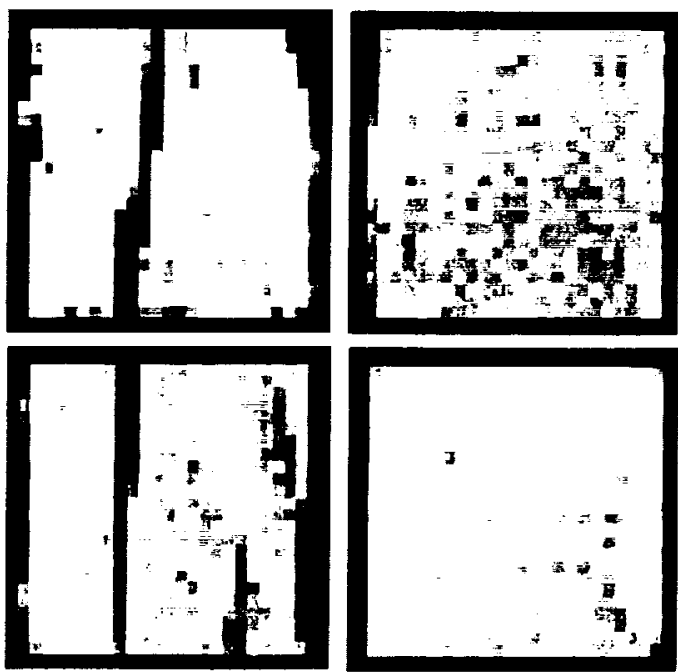

$1 \mathrm{~cm}$

Figure 6. (a) IR transmission image of $\mathrm{eV}$ Products specimen 1185 showing two grain boundaries decorated with tellurium inclusions. (b) X-ray spectral map (counts in peak channel) of eV Products specimen 1185-1 showing poor detector response in the regions of the decorated grain boundaries. 
Twin boundaries decorated with a lower density of tellurium inclusions, which were present in the set of nine larger specimens, did not produce degradation in detector performance when mapped using a $500 \mu \mathrm{m}$ collimator. Others have reported that decorated twins as well as tilt boundaries degrade detector performance. ${ }^{7.8}$ The effects of voids, which are in the form of pipe, depend on several factors including the proximity to the surface and the proximity to other pipes. Lone pipes located near the mid-plane of a specimen have little or no effect on detector performance. Pipe located near one surface and groups of pipe with various through-the-thickness positions can produce poor detector performance. Dispersed telluride inclusions appear to have no deleterious effects on detector performance. This finding has also been previously reported,' and is not surprising because of the small size of the inclusions (approximately 5 to $10 \mu \mathrm{m}$ ) compared to the size of the collimated beam $(100-500 \mu \mathrm{m})$.

\subsection{Wafer Scale Mapping}

Even with the high photon flux provided by the $\mathrm{x}$-ray tube, the estimated time required to perform spectral mapping of an entire 5 inch diameter CdZnTe wafer at a $500 \mu \mathrm{m}$ resolution is 80 hours. Based on the population densities of typical bulk defects in CdZnTe, a large portion of this time would be spent mapping regions with no chance of yielding large area detectors. Our correlation data suggests that an initial IR transmission screening of an entire wafer can identify areas which have the best potential for yielding large defect free specimens. In essence, IR transmission imaging could be used to generate mining maps.

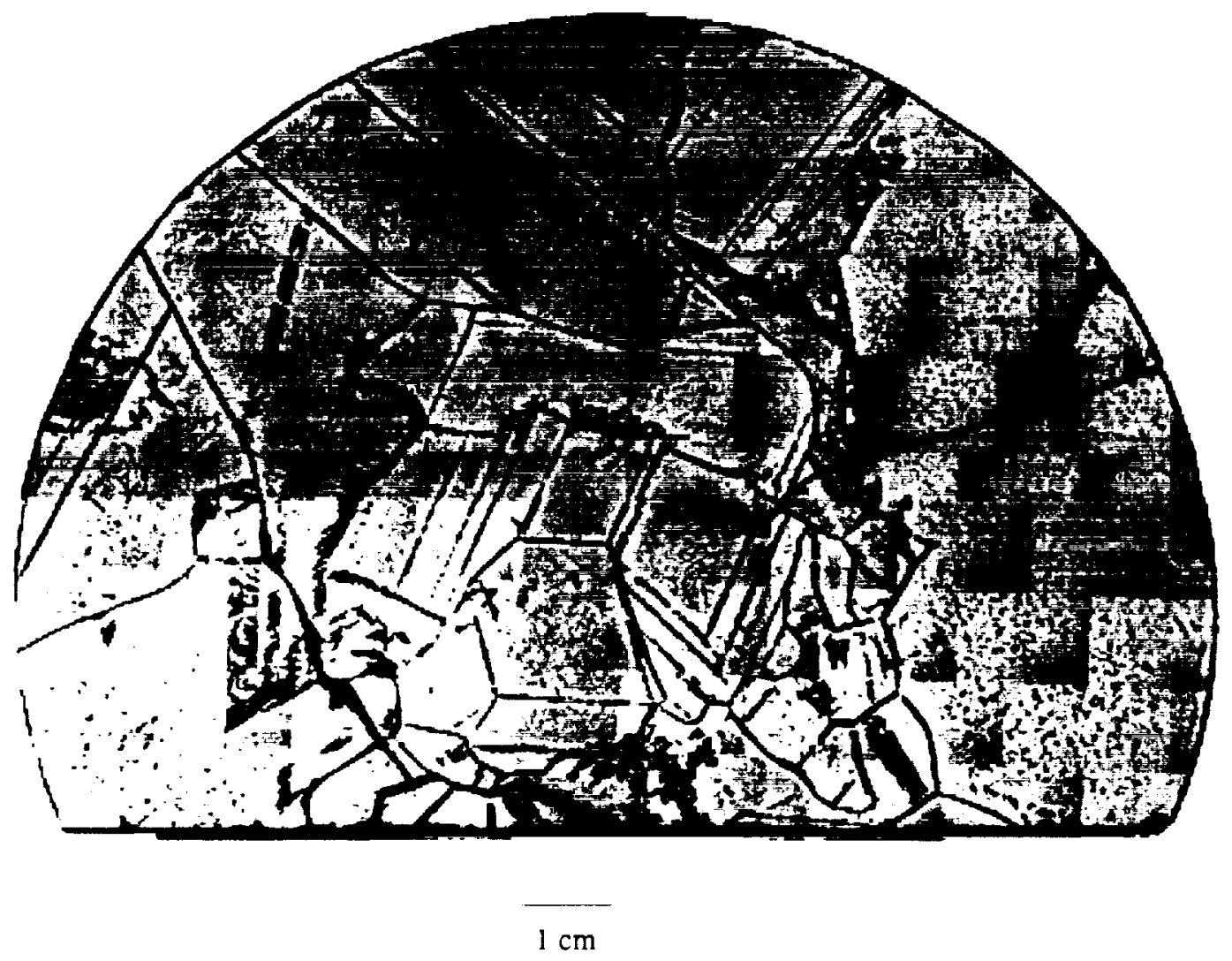

Figure 7. IR transmission image of an wafer slice from an ingot. 
In order to help us test this hypothesis, eV Products supplied numerous $2 \mathrm{~mm}$ thick polished CdZnTe wafer slices that were cut perpendicular to the ingot growth direction. We performed IR transmission imaging on the slices to locate areas that could yield uniform 26.9 by $26.9 \mathrm{~mm}$ specimens. Figure 7 shows a typical IR image of a wafer slice. In order to increase the yield, defects located near the comers of a specimen were deemed acceptable, as the instrument application uses a circular detection area approximately centered on the square detector.
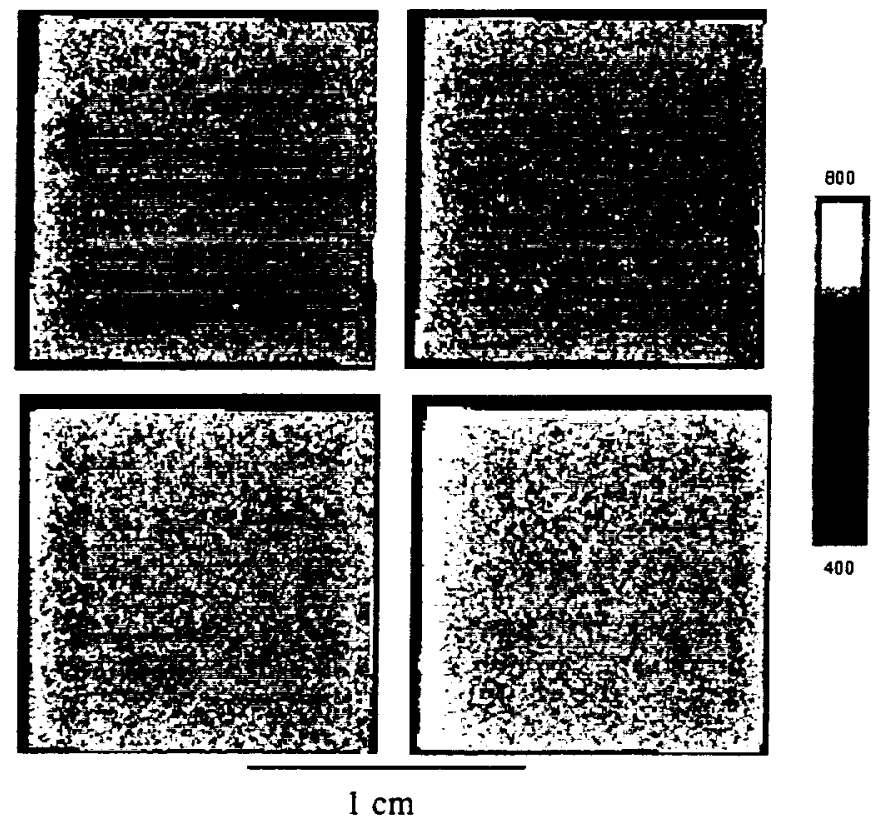

Figure 8. X-ray spectral map of a specimen mined from a wafer slice based on IR transmission images. The plots of counts in the peak channel show good uniformity in spectroscopic response.

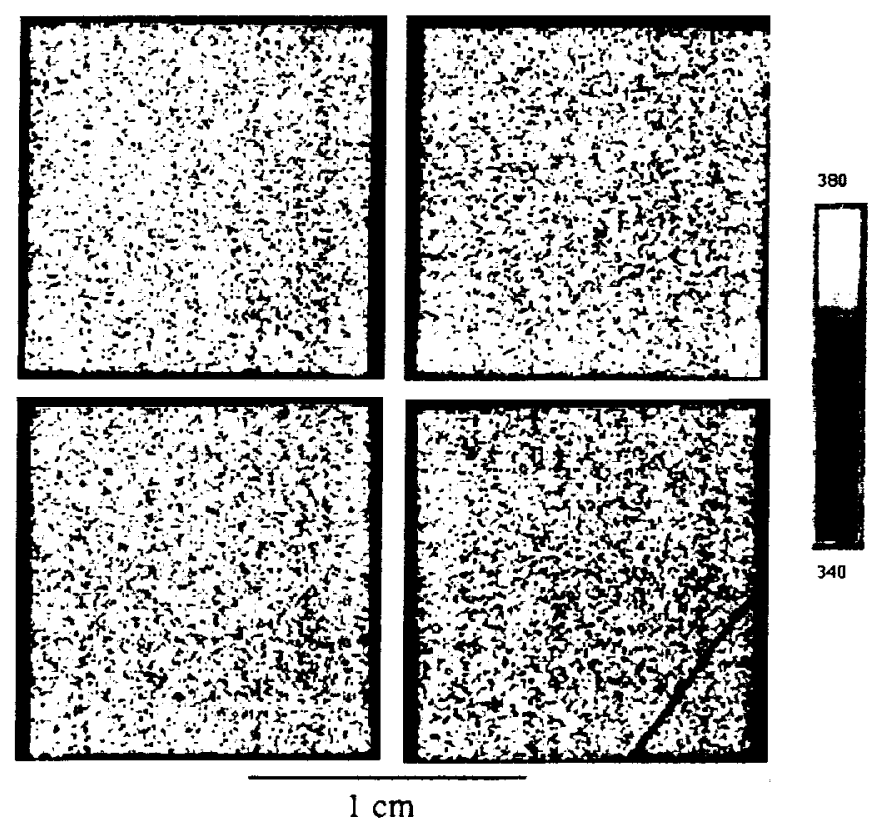

Figure 9. X-ray spectral map of a specimen mined from a wafer slice based on IR transmission images. The plots of the peak channel show good uniformity in spectroscopic response. The line in the lower right quadrant corresponds to a decorated grain boundary. 
The first nine wafer slices, which came from two different ingots, yielded nine locations for mining. All nine of the specimens came from one set of four slices, and one slice from this set yielded three specimens. The remaining set of five wafer slices yielded no specimens. During the cutting of the specimens from the slices, one was lost due to cracking at a grain boundary near a comer. The remaining eight specimens were returned for $\mathbf{x}$-ray spectral mapping. The initial mapping was performed using a $250 \mu \mathrm{m}$ collimator and $250 \mu \mathrm{m}$ step size. Again, the specimens were configured with four pixels and a guard ring for the mapping. Based on the mapping results, seven of the eight specimens were purchased from $\mathrm{eV}$ Products. This is a dramatic increase in the yield of large area specimens.

We are now in the process of mapping the purchased specimens using a $100 \mu \mathrm{m}$ collimator and $100 \mu \mathrm{m}$ step size so that the very best pieces can be selected for the flight detectors. Figure 8 shows the high resolution map from one specimen with a uniform spectroscopic response. Figure 9 shows a specimen with a comer grain which was detected in the IR and deemed to be outside the critical detection area. This specimen also contained three decorated twin boundaries which transversed the entire specimen width. The twins produced no degradation in spectroscopic response. These results show that IR imaging can be used to successfully identify areas within wafer slices that will produce uniform detector response.

\section{CONCLUSIONS}

IR transmission images of wafer slices of $\mathrm{CdZnTe}$ from $\mathrm{eV}$ Products have been successfully used to identify large area detector specimens with uniform spectroscopic response. The IR images readily detect grain boundaries decorated with tellurium inclusions, which are the most detrimental bulk defects in high pressure Bridgeman CdZnTe obtained from eV Products. The other two types of bulk defects in the wafer slices, decorated twins and dispersed tellurium inclusions, had no detrimental effect on detector performance at spatial resolutions as low as $100 \mu \mathrm{m}$. We have also demonstrated that a $\mathrm{x}$-ray tube can be used as a source of photons for $x$-ray spectroscopic mapping. The key advantage of using a $x$-ray tube is the high photon flux, which translates to short counting times.

\section{ACKNOWLEDGEMENTS}

Funding for this research has been provided by the NASA Office of Safety \& Mission Assurance. We also acknowledge the efforts of $\mathrm{eV}$ Products, who provided the polished wafer slices from which specimens were mined.

\section{REFERENCES}

1. N. Gehrels and J. Paul, "THE NEW GAMMA-RAY ASTRONOMY," Physics Today, 26-32, February 1998.

2. A. Parsons, D. M.Palmer, P. Kurczynski, L. Barbier, S. Barthelmy, L. Bartlett, N. Gehrels,J. Krizmanic, C. M. Stahle, J. Tueller, and B. Teegarden, "PERFORMANCE OF PROTOTYPE SEGMENTED CdZnTe ARRAYS,"," Semiconductors for Room-Temperature Radiation Detector Applications II, R.B. James, T.E. Schlesinger, P. Siffert, W. Dusi, M.R. Squillante, M. O'Connell, and M. Cuzin, Vol. 487, 147-152, Materials Research Society, Warrendale, PA, 1998.

3. C.M. Stahle, B.H. Parker, A.M. Parsons, L.M. Barbier, S.D. Barthelmy, N.A. Gehrels, D.M. Palmer, S.J. Snodgrass, and J. Tueller, "CdZnTe and CdTe Detector Arrays for Hard X-Ray and Gamma-Ray Astronomy," accepted for publication in Nucl. Inst. Meth. Phys. Res. A, 1999.

4. K. Yamashita, P.J. Serlemitsos, J. Tueller, S.D. Barthelmy, ,L.M. Bartlett, K.-W. Chan, A. Furuzawa, N. Gehrels, K. Haga, H. Kunieda, P. Kurczynski, G. Lodha, N. Nakajo, N. Nakamura, Y. Ogasaka, T. Okajima, D. Palmer, A. Parsons, Y. Soong, C.M. Stahle, H. Takata, K. Tumura, Y. Tawara, and B. Teegarden, "Supermirror hard X-ray telescope," Applied Optics, Vol. 37, 1-7, December 1998.

5. J.M. Woolfenden, H.B. Barber, H.H. Barrett, E.L. Dereniak, J.D. Eskin, D.G. Marks, K.J. Matherson, E.T. Young, and F.L. Augustine, "MODULAR 64X64 CdZnTe ARRAYS WITH MULTIPLEXER READOUT FOR HIGHRESOLUTION NUCLEAR MEDICINE IMAGING," Semiconductors for Room-Temperature Radiation Detector Applications II, R.B. James, T.E. Schlesinger, P. Siffert, W. Dusi, M.R. Squillante, M. O'Connell, and M. Cuzin, Vol. 487, 239-243, Materials Research Society, Warrendale, PA, 1998. 
6. F.P Doty, J.P. Cozzatti and J.P. Schomer, "Characterization of Bulk CdZnTe by IR Transmission Imaging," Hard $X-R a y$ and Gamma-Ray Detector Physics, Optics, and Applications, R.B. Hoover, F.P. Doty, Vol 3115, 51-55, SPIE-The International Society for Optical Engineering, Bellingham, WA, 1997.

7. C. Szeles and E.E. Eissler, "CURRENT ISSUES OF HIGH-PRESSURE BRIDGEMAN GROWTH OF SEMIINSULATING CdZnTe," Semiconductors for Room-Temperature Radiation Detector Applications II, R.B. James, T.E. Schlesinger, P. Siffert, W. Dusi, M.R. Squillante, M. O'Connell, and M. Cuzin, Vol. 487, 3-12, Materials Research Society, Warrendale, PA, 1998.

8. H. Yoon, J.M. Van Scyoc, T.S. Gilbert, M.S. Goorsky, B.A. Brunett, J.C. Lund, H. Hermon, M. Schieber, and R.B. James, "INFLUENCE OF STRUCTURAL DEFECTS AND ZINC COMPOSITION VARIATION ON THE DEVICE RESPONSE OF $\mathrm{Cd}_{1-\mathrm{x}} \mathrm{Zn}_{\mathrm{x}}$ Te RADIATION DETECTORS," Semiconductors for Room-Temperature Radiation Detector Applications II, R.B. James, T.E. Schlesinger, P. Siffert, W. Dusi, M.R. Squillante, M. O'Connell, and M. Cuzin, Vol. 487, 115-120, Materials Research Society, Warrendale, PA, 1998.

9. B.A. Brunett, J.M. Van Scyoc, H. Yoon, T.S. Gilbert, T.E. Schlesinger, J.C. Lund, R.B. James, "MAPPING OF LARGE AREA CADMTUM ZIN TELLURIDE (CZT) WAFERS: APPARATUS AND METHODS," Semiconductors for Room-Temperature Radiation Detector Applications II, R.B. James, T.E. Schlesinger, P. Siffert, W. Dusi, M.R. Squillante, M. O'Connell, and M. Cuzin, Vol. 487, 39-44, Materials Research Society, Warrendale, PA, 1998.

10. C.M. Stahle, Z.Q. Shi, K. Hu, S.D. Barthelmy, S.J. Snodgrass, L.M. Bartlett, P.K. Shu, S.J. Lehtonen and K.J. Mach, "Fabrication of CdZnTe strip detectors for large area arrays," ," Hard X-Ray and Gamma-Ray Detector Physics, Optics, and Applications, R.B. Hoover, F.P. Doty, Vol 3115, 90-97, SPIE-The International Society for Optical Engineering, Bellingham, WA, 1997. 